\title{
Human Fallopian Tube Mesenchymal Stromal Cells Enhance Bone Regeneration in a Xenotransplanted Model
}

\author{
Tatiana Jazedje • Daniela F. Bueno $\cdot$ Bruno V. P. Almada $\cdot$ Heloisa Caetano • \\ Carlos E. Czeresnia • Paulo M. Perin - Silvio Halpern - Mariangela Maluf • \\ Lucila P. Evangelista • Marcelo G. Nisenbaum • Marília T. Martins • \\ Maria R. Passos-Bueno • Mayana Zatz
}

Published online: 9 July 2011

(C) The Author(s) 2011. This article is published with open access at Springerlink.com

\begin{abstract}
We have recently reported that human fallopian tubes, which are discarded during surgical procedures of women submitted to sterilization or hysterectomies, are a rich source of human fallopian tube mesenchymal stromal cells (htMSCs). It has been previously shown that human mesenchymal stromal cells may be useful in enhancing the speed of bone regeneration. This prompted us to investigate whether htMSCs might be useful for the treatment of osteoporosis or other bone diseases, since they present a pronounced capacity for osteogenic differentiation in vitro. Based on this prior knowledge, our aim was to evaluate, in vivo, the osteogenic capacity of htMSCs to regenerate bone through an already described xenotransplantation model: nonimmunosuppressed (NIS) rats with cranial defects. htMSCs were obtained from five 30-50 years old healthy women and characterized by flow cytometry and for their
\end{abstract}

Tatiana Jazedje and Daniela F. Bueno contributed equally for this work.

T. Jazedje $\cdot$ D. F. Bueno $\cdot$ B. V. P. Almada $\cdot$ H. Caetano $\cdot$

M. R. Passos-Bueno $\cdot$ M. Zatz $(\square)$

Human Genome Research Center, Biosciences Institute,

University of São Paulo,

São Paulo, Brazil

e-mail: mayazatz@usp.br

C. E. Czeresnia $\cdot$ S. Halpern • L. P. Evangelista $・$ M. G. Nisenbaum Célula Mater,

São Paulo, Brazil

P. M. Perin • M. Maluf

CEERH Specialized Center for Human Reproduction, São Paulo, Brazil

M. T. Martins

Department of Oral Pathology, University of São Paulo,

São Paulo, Brazil multipotenciality in vitro capacity (osteogenic, chondrogenic and adipogenic differentiations). Two symmetric fullthickness cranial defects on each parietal region of seven NIS rats were performed. The left side (LS) of six animals was covered with CellCeram (Scaffdex) - a bioabsorbable ceramic composite scaffold that contains $60 \%$ hydroxyapatite and $40 \% \beta$-tricalciumphosphate - only, and the right side (RS) with the CellCeram and htMSCs $\left(10^{6}\right.$ cells/scaffold $)$. The animals were euthanized at 30,60 and 90 days postoperatively and cranial tissue samples were taken for histological analysis. After 90 days we observed neobone formation in both sides. However, in animals euthanized 30 and 60 days after the procedure, a mature bone was observed only on the side with htMSCs. PCR and immunofluorescence analysis confirmed the presence of human DNA and thus that human cells were not rejected, which further supports the imunomodulatory property of htMSCs. In conclusion, htMSCs can be used successfully to enhance bone regeneration in vivo, opening a new field for future treatments of osteoporosis and bone reconstruction.

Keywords Fallopian tube - Mesenchymal stromal cells . Bone regeneration $\cdot$ Regenerative medicine $\cdot$ Xenotransplant

\section{Introduction}

Osteoporosis is a common skeletal condition, characterized by a decrease in bone's density and increased risk of fractures. Its prevalence is much higher in postmenopausal women than in older men, and may affect about $40-70 \%$ of women over 70 years old. Although osteoporosis is still under-diagnosed and under-treated, the risk of fracture is high and is associated to morbidity, especially when it occurs in the spine and hip 
$[1,2]$. Recent reports show that the use of mesenchymal stromal cells (MSCs) isolated from adult organs and tissues is becoming a very attractive strategy for the treatment of fractures and other bone disorders such as congenital anomalies, traumas and cancer [3-5].

Adult MSCs are typically defined as undifferentiated multipotent cells endowed with the capacity for selfrenewal and the potential to differentiate into several distinct cell lineages [6]. These progenitor cells, which constitute a reservoir found within the connective tissue of most organs, are involved in the maintenance and repair of tissues throughout the postnatal life of an individual.

Functionally heterogeneous, MSCs populations isolated from different tissues present a similar profile of cell surface receptor expression. It is also well known that adult stem cells are defined by their functional properties rather than by marker expression. We and others have recently shown that umbilical cord, dental pulp, orbicular oris muscle, adipose tissue and fallopian tubes are a very rich source of MSCs able to differentiate into muscle, cartilage, bone and adipose cell lineages [7-11].

Bone is a tissue of constant remodeling and MSCs might play an important role in this process, therefore contributing to treat osteoporosis or other bone diseases. In addition, the possibility to use autologous stem cells, from discarded surgical tissues is of great interest. We have previously shown that human fallopian tube mesenchymal stromal cells (htMSCs) display great osteogenic differentiation in vitro [11], but we have also observed that MSCs may differ in their in vitro differentiation potential when injected in animal models [12]. htMSCs could represent an additional autologous source of MSCs for bone repair for those women that had their fallopian tube previously removed surgically, since htMSCs are a cell population that can be rapidly expanded for potential clinical applications [11]. Furthermore, their pronounced capacity for osteogenic differentiation in vitro prompted us to evaluate the osteogenic capacity of htMSCs to regenerate bone in vivo. However, prior to start clinical trials, it is of paramount importance to test whether it is possible to make bone tissue engineering in vivo, using htMSCs. Therefore, the aim of the present study was to evaluate, in vivo, the osteogenic capacity of htMSCs to regenerate bone when xenotransplanted to non-immunosuppressed (NIS) rats with cranial defects, as already described before with MSCs from different origins $[8,9]$.

\section{Material and Methods}

Human Fallopian Tube Collection and Processing

Human fallopian tubes $(n=5)$ were obtained from hysterectomy or tubal ligation/resection samples collected from women aged 30-50 years who had not undergone exogenous hormonal treatment for at least 3 months prior to surgery. Furthermore, all donors were considered fertile, since they had children by natural conception. Informed consent was obtained from each patient and approval granted from the ethics committee of the Biosciences Institute of the University of São Paulo. All laboratory experiments were carried out at the Human Genome Research Center, São Paulo, Brazil.

All steps of collection and processing were previously described [11]. All samples that had been kept frozen in liquid nitrogen were thawed and expanded until the 4th passage, characterized by flow cytometry and differentiation potential in vitro, before the in vivo experiments.

\section{Flow Cytometry Analysis}

Flow cytometry analysis was performed using a Guava EasyCyte microcapillary flow cytometer (Guava Technologies, Hayward, CA) utilizing laser excitation and emission wavelengths of 488 and $532 \mathrm{~nm}$, respectively. Cells were pelleted, resuspended in PBS (GibcoInvitrogen, Carlsbad, CA) at a concentration of $1.0 \times 10^{5}$ cells $/ \mathrm{mL}$ and stained with saturating concentration of antibodies. After $45 \mathrm{~min}$ incubation in the dark at room temperature, cells were washed three times with PBS (Gibco, Invitrogen, Carlsbad, CA) and resuspended in $0.25 \mathrm{~mL}$ of cold PBS.

In order to analyze cell surface expression of protein markers, adherent cells were treated with the following anti-human primary antibodies: CD13-phycoerythrin [PE] (Becton Dickinson, Franklin Lakes, NJ), CD29-PE-Cy5, CD31-PE, CD34-PerCP, CD90-R-PE, human leukocyte antigens (HLA)-ABC-FITC and HLA-DR-R-PE (Becton Dickinson, Franklin Lakes, NJ), SSEA3 (Chemicon, Temecula, CA), STRO1 (R\&D Systems, Minneapolis, $\mathrm{MN}$ ), and $\mathrm{SH} 3$ and (kindly provided by Dr. Kerkis, Butantan Institute, São Paulo, Brazil). Unconjugated markers were reacted with anti-mouse PE secondary antibody (Guava Technologies, Hayward, CA). Unstained cells were gated on forward scatter to eliminate particulate debris and clumped cells. A minimum of 5.000 events were counted for each sample.

\section{Mesenchymal Stromal Cells Differentiation}

To evaluate the properties of mesenchymal stromal cells differentiation, adherent cells (4th passages) underwent in vitro adipogenic, chondrogenic and osteogenic differentiations. Invitrogen Stem Pro differentiation medium kits (A1007101, A1007001 and A1007201), prepared according to manufacturer data sheets were used. 


\section{Adipogenic}

Cells were kept in culture for 2 weeks. Confirmation of adipogenic differentiation was obtained on day 21 by intracellular accumulation of lipid-rich vacuoles stainable with oil red O (Sigma-Aldrich, St. Louis, MO). For the oil red $\mathrm{O}$ stain, cells were fixed with $4 \%$ paraformaldehyde (PFA) for $30 \mathrm{~min}$, washed, and stained with a working solution of $0.16 \%$ oil red $\mathrm{O}$ for $20 \mathrm{~min}$.

\section{Chondrogenic}

After 2 weeks of culture, monolayer cells were fixed with 4\% PFA for 10 min and dyed with Toluidine Blue, which stains extracellular matrix mucopolysaccharides. Staining solution was prepared by adding $1 \%$ of Toluidine Blue dissolved in distilled water containing $1 \%$ of sodium borate, followed by filtering. Solution was added to each culture well for 2 min and then washed with distilled water and left to air-dry.

\section{Osteogenic}

Osteogenic differentiation was shown by formation of calcium-hydroxyapatite-positive areas (von Kossa staining) on day 21. After two washes with PBS (GibcoInvitrogen, Carlsbad, CA) and one with distilled water, the cells were incubated in 1\% silver nitrate (Sigma- Aldrich, St. Louis, MO) under ultraviolet light for $45 \mathrm{~min}$. The cells were then incubated in $3 \%$ sodium thiosulfate (Sigma-Aldrich, St. Louis, MO) for $5 \mathrm{~min}$. Counterstaining was finally performed with Van Gieson stain. The calcium accumulation was indicated by dark color. Alizarin Red S staining, which is incorporated by calcium-containing mineralized matrix, was also provided for each cell line after 21 days of differentiation as well. The methodology is described in the osteogenic kit data sheet (A1007201/Invitrogen).
Animal Model

The present study was approved by the Animal Research Ethics Committee at the Institute of Biosciences, University of São Paulo. A total of seven NIS Wistar rats (3-month-old males, body weight $320-420 \mathrm{~g}$ each) were selected. The animals were anesthetized with intraperitoneal injections $(0.3 \mathrm{~mL}=100 \mathrm{~g}$ body weight $)$ containing ketamine hydrochloride $(5 \%)$ and xylazine $(2 \%)$. The rats were then positioned in a cephalostat during the procedure. A midline skin incision from the nasofrontal area to the external occipital protuberance was performed. The skin and underlying tissues were reflected laterally to expose the full extent of the calvaria. Two symmetrical full-thickness cranial defects with $4.5 \mathrm{~mm}$ of diameter were made on each parietal region using a micromotor drill under constant irrigation with sterile physiologic solution to prevent bone overheating. The duramater was maintained intact. The left side (LS) of six animals was covered with CellCeram scaffold (Scaffdex, ref CM0002) only-a bioabsorbable ceramic composite scaffold that contains $60 \%$ hydroxyapatite and $40 \% \beta$-tricalciumphosphateand the right side (RS) with the CellCeram and htMSCs $\left(10^{6}\right.$ cells/scaffold $)$. In one control animal, the RS had CellCeram only and nothing was added at the left side. Scaffolds with htMSCs were prepared $24 \mathrm{~h}$ before the surgeries. Firstly, cells were added on the surface of the scaffold with $100 \mu \mathrm{l}$ of culture medium (in a $35 \mathrm{~mm}$ cell culture dish) and kept in culture for $1 \mathrm{~h}$. After that, $2 \mathrm{ml}$ of medium was added and the scaffold with cells was kept in culture overnight, until use. Animals were euthanized at 30, 60 and 90 days postoperatively and cranial tissue samples were taken for histological analysis.

\section{Electron Microscopy}

Cells were fixed for $24 \mathrm{~h}$ at $4^{\circ} \mathrm{C}$ using the modified Karnovsky solution, which contains $2.5 \%$ glutaraldehyde,
Table 1 Cytometry analysis of htMSCs. Analyzed markers, commitment and expression (the percentage of positive labeled cells). (GuavaTechnologies, Hayward, CA, http://www. guavatechnologies.com)

\begin{tabular}{|c|c|c|c|c|c|c|}
\hline $\begin{array}{l}\text { htMSCs } \\
\text { Marker }\end{array}$ & $\begin{array}{l}\text { Lineages } \\
\text { Commitment/Relevance }\end{array}$ & \multicolumn{5}{|c|}{$\%$ Labeled cells } \\
\hline CD34 & Hematopoietic SC factor & 1.78 & 2.62 & 1.16 & 1.30 & 0.80 \\
\hline CD31 & Endothelial & 0.68 & 0.44 & 2.90 & 0.43 & 1.85 \\
\hline HLA-DR & HLA & 0.64 & 0.65 & 3.80 & 0.53 & 1.58 \\
\hline HLA-ABC & HLA & 95.55 & 95.55 & 96.89 & 97.66 & 99.15 \\
\hline CD90 & MSC/adherent marker & 99.26 & 99.42 & 98.87 & 99.76 & 98.85 \\
\hline STRO1 & MSC marker & 2.12 & 9.60 & 8.75 & 2.29 & 2.41 \\
\hline SSEA3 & Embryonic stem cell & 0.09 & 0.46 & 3.81 & 0.42 & 0.70 \\
\hline $\mathrm{SH} 3$ & MSC marker & 98.58 & 98.56 & 99.43 & 98.07 & 98.77 \\
\hline CD13 & MSC marker & 98.44 & 98.24 & 98.89 & 98.24 & 99.50 \\
\hline CD29 & MSC/adherent marker & 98.81 & 98.24 & 96.92 & 98.78 & 99.45 \\
\hline
\end{tabular}



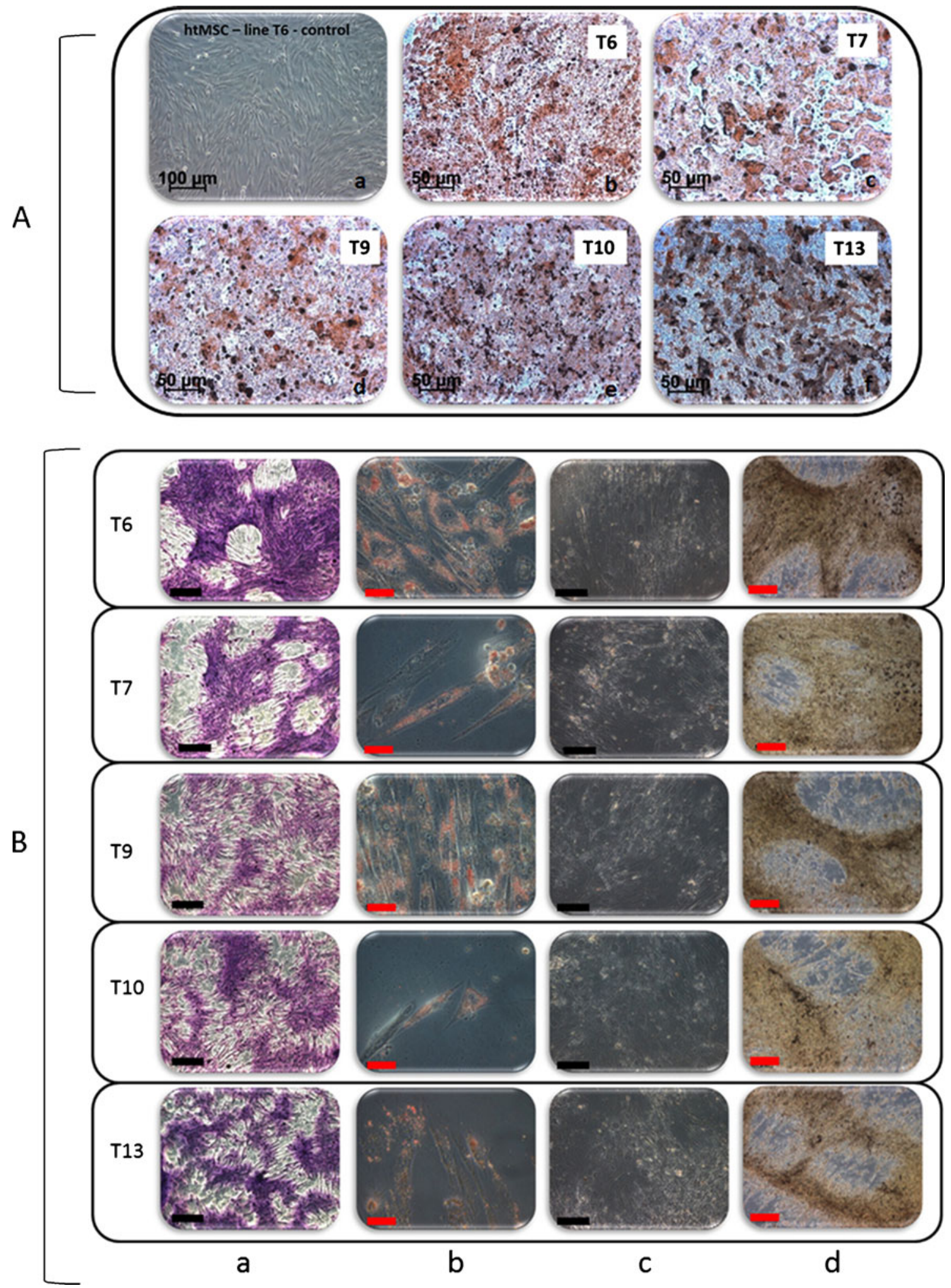

Fig. 1 Multilineage differentiation in vitro. Panel a: $a$ Example of undifferentiated htMSC line (T6). $b, c, d, e$ and $f$ Osteogenic differentiation after 3 weeks of induction for each cell line (T6, T7, T9, T10, T13 respectively)-Alizarin S Red staining. Panel b: Each cell line is represented in a row (T6, T7, T9, T10 and T13). Column $a$
Chondrogenic differentiation-Toluidine Blue staining $(50 \times)$. Column $b$ Adipogenic differentiation-Oil Red staining $(400 \times)$. Columns $c$ and $d$ Osteogenic differentiation after $2(c)$ and $3(d)$ weeks of induction-Von Kossa staining (200× and 100×). Black scale bar: $500 \mu \mathrm{m}$; Red scale bar: $100 \mu \mathrm{m}$ (Microscope Zeiss Axiovert 200) 
$2 \%$ paraformaldehyde in $0.1 \mathrm{M}(\mathrm{pH} 7.4)$ sodium cacodilate buffer. The specimens were post fixed in $2 \%$ osmium tetroxide solution, rinsed in distilled water for $1 \mathrm{~h}$ at room temperature. Then, the cells were dehydrated in an increasing series of ethanol and cleaned in an ultrasonic apparatus (Gerador DA 200, Thornton Inpec Eletronica SA, São Paulo, SP, Brazil) for $1 \mathrm{~h}$. The samples were mounted on metal stubs and covered with gold in a sputter coater apparatus (Balzers Union SCD-040, Liechtesnstein). The specimens were examined in a scanning electron microscope at $20.0 \mathrm{kV}$ (Jeol 6460LV, Tokyo, Japan).

Histological Preparation

\section{Hematoxylin and Eosin (HE)}

Sections from the 30th, 60th and 90th postsurgery days were obtained for histological assessment. Tissue samples were fixed in $10 \%$ formalin solution for $24 \mathrm{~h}$, decalcified in $5 \%$ formic acid for $48 \mathrm{~h}$, and paraffin embedded. For morphological study, 5-mm sections stained with hematoxylin and eosin (HE) were examined under an AxioVert 200 microscope (Axio Imager Z1, Carl Zeiss, Oberkochen, Germany).

\section{Human DNA Amplification}

To assess the presence of human cells in the new bone tissue from the left side (scaffold only) and right side (scaffold with htMSCs) from a rat euthanized 90 days after transplantation we performed DNA extraction through QIAamp DNA Mini KIT (Qiagen, Venlo, The Netherlands). After that, PCR reactions were performed with specific human amelogenin gene primers [13].

\section{Immunohistochemistry}

The sections were deparaffinized with two 5-min washes in xylene, hydrated in graded ethanol series and then rinsed in distilled water. The samples were fixed for $20 \mathrm{~min}$ at room temperature with $4 \%$ paraformaldehyde in PBS and washed three times with $100 \mathrm{mM}$ glycine in PBS. For antigen retrieval, slides were incubated for $40 \mathrm{~min}$ in citrate buffer and then cooled for $20 \mathrm{~min}$ at room temperature, rinsed in phosphate buffered saline (PBS), and blocked for $1 \mathrm{~h}$ in immunofluorescent blocking buffer (IBB-5\% BSA, 10\% FBS, $1 \mathrm{X}$ PBS, and $0.1 \%$ Triton X-100). Samples were then incubated for $1 \mathrm{~h}$ at room temperature with 1:100 mouse anti-human nuclei monoclonal antibody ( $\mathrm{HuNu}$; Chemicon, Temecula, CA), washed with PBST, and incubated with secondary antibody (1:600 Alexa Fluor 456 anti-mouse $\mathrm{IgG}$; Invitrogen) for $1 \mathrm{~h}$ at room temperature. Slides were examined using an Axiovert 200 microscope (Axio Imager Z1, Carl Zeiss, Oberkochen, Germany).

\section{Results}

Flow Cytometry Analysis

The five lineages of htMSCs chosen for the experiments did not express the hematopoietic lineage marker CD34 and the endothelial marker CD31. In addition, the majority of cells expressed high levels of adhesion markers (CD29 and CD90) and MSCs markers (CD13, SH3 and SSEA3). Cells were also positive for HLA-class I (HLA-ABC) but negative

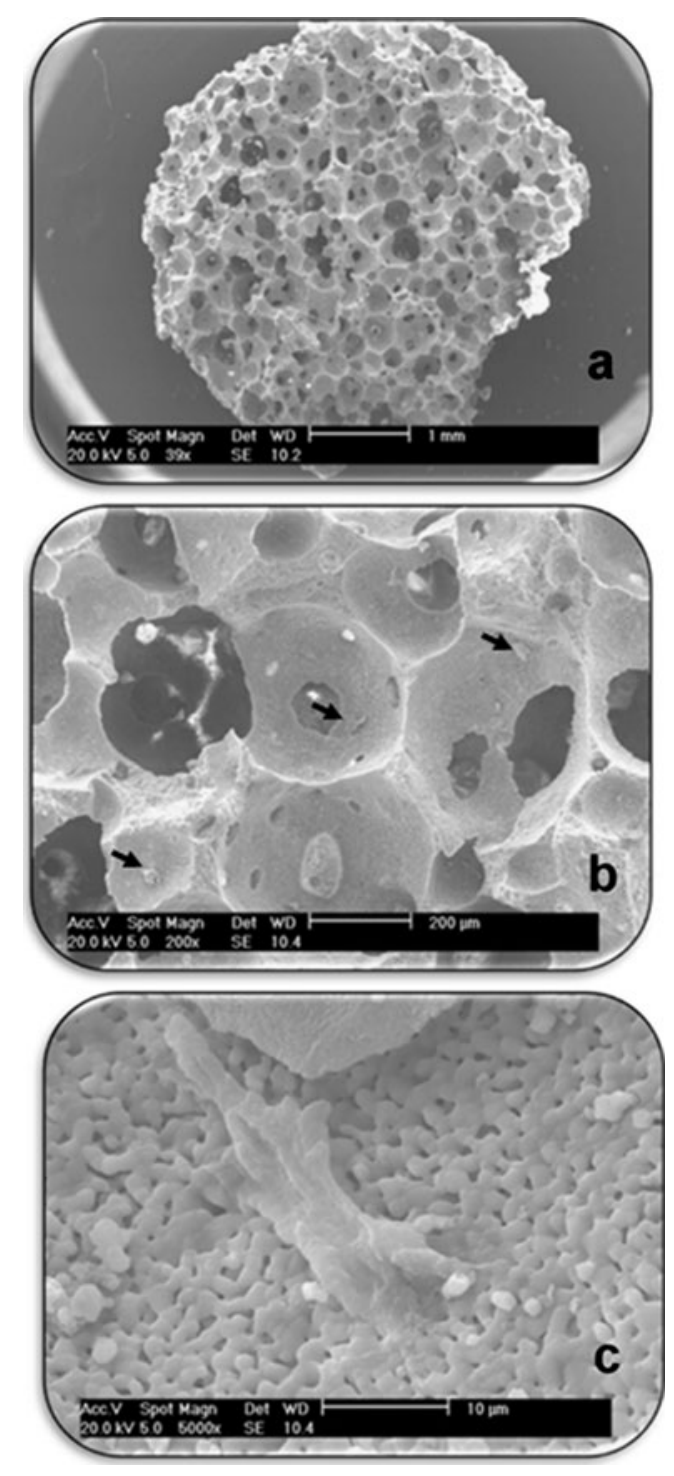

Fig. 2 Electron microscopy. Scaffold seeded with htMSC. a Entire scaffold b Visual approach to identification of the cells (arrows) c htMSC in detail, attached onto scaffold 
for HLA-class II (HLA-DR), as well for the presumed MSCs marker Stro1 (Table 1).

\section{Multilineage Differentiation}

The plasticity of each chosen lineage of htMSCs was assessed 2 weeks after mesodermal induction for adipogenic and chondrogenic differentiation. In order to confirm the good osteogenic capacity of each chosen htMSCs line, osteogenic differentiation was assessed 2 and/or 3 weeks after mesodermal induction. The htMSCs differentiated in adipogenic, chondrogenic, and osteogenic tissues in vitro (Fig. 1), confirming their mesenchymal nature and their multipotent potential.

\section{Electron Microscopy}

Electron microscopy analysis confirmed the attachment of htMSCs onto the scaffolds (Fig. 2).
Histological Analysis

\section{In Vivo Osteogenic Potential of htMSCs}

We have evaluated the in vivo osteogenic potential of htMSCs using a previously established model $[8,9]$. None of the animals died of infection or any other complication as a result of surgery or cell transplantation process. Histological examination of the cranial defect 30 days postsurgery in two animals revealed intense deposition of new bone on the right side, with CellCeram and $10^{6}$ htMSCs (Fig. $3 b$ and $b^{\prime}$ ). On the other hand, the side containing only CellCeram had the defect filled with loose connective tissue exhibiting chronic inflammatory infiltrate, intermingled with remnants of the scaffold (Fig. 3a and a'). At 60 days postsurgery, a histological evaluation of the right side, which contained both the htMSCs and the CellCeram, revealed reduction of granulation tissue, and the bone was in an advanced maturation stage, with some lamellae formation

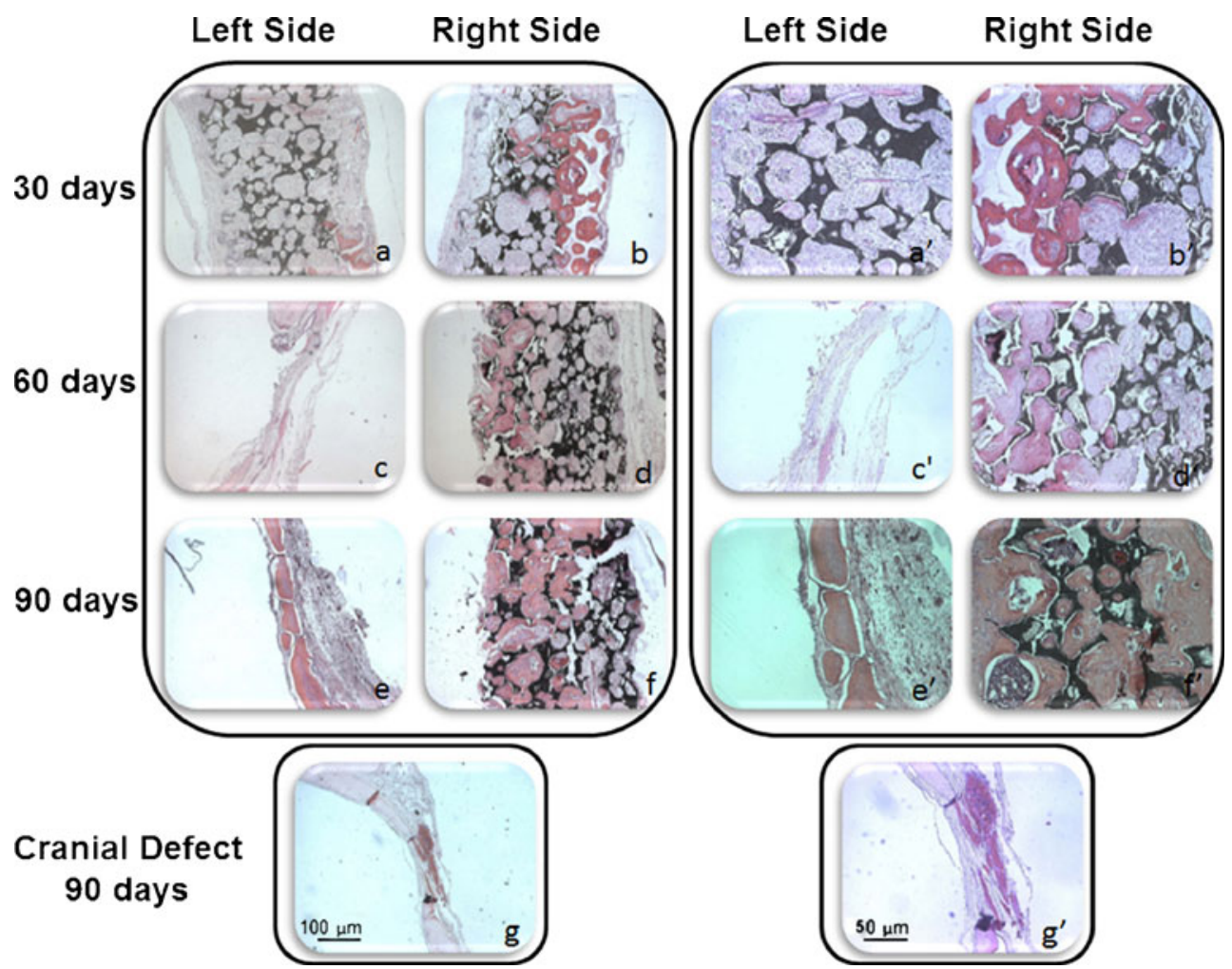

Fig. 3 In vivo osteogenic potential of htMSCs. a and b 30 days postsurgery; a represents the left side, without stem cells on the scaffolds, were neobone formation was not observed; b represents the right side, with htMSCs, where it's possible to perceive an intense deposition of new bone. $\mathbf{a}^{\prime}$ and $\mathbf{b}^{\prime}$ The same histological slides in higher magnification. c and d 60 days postsurgery; c represents the left side, without stem cells on the scaffolds, were neobone formation was not observed, but there was reabsorption of the biomaterial; $\mathbf{d}$ represents the right side, with htMSCs, where there is bone in an advanced maturation stage. $\mathbf{c}^{\prime}$ and $\mathbf{d}^{\prime}$ The same histological slides in higher magnification. e and f 90 days postsurgery; e represents the left side, without stem cells on the scaffolds, where it is possible to see neobone formation onto the reabsorbed scaffold local; f represents the right side, with htMSCS, where it is possible to see a larger amount of neobone. $\mathbf{e}^{\prime}$ and $\mathbf{f}^{\prime}$ The same histological slides in higher magnification. g Control animal, with cranial defect, 90 days postsurgery. $\mathbf{g}^{\prime}$ The same histological slide in higher magnification 
in both studied animals (Fig. $3 \mathrm{~d}$ and $\mathrm{d}^{\prime}$ ). On the left side, with CellCeram only, no neobone formation was observed and there was a complete reabsorption of the scaffold (Fig. 3c and $\mathrm{c}^{\prime}$ ). At 90 days postsurgery, we observed neobone formation in both sides, but the right side presented visually a larger amount of neobone (Fig. 3e, e'-left side, Fig. 3f and $\mathrm{f}^{\prime}$-right side). One control animal, with the cranial defect (without scaffold and/or stem cells) was kept for 90 days. In this animal there was no spontaneous skull regeneration of the cranial defect up to 12 weeks (Fig. $3 \mathrm{~g}$ and $\mathrm{g}^{\prime}$ ) as illustrated by histological analysis.

In the experimental group, a positive staining for human nuclei through immunohistochemical analysis was observed only on the right side of two animals 90 days postsurgery.

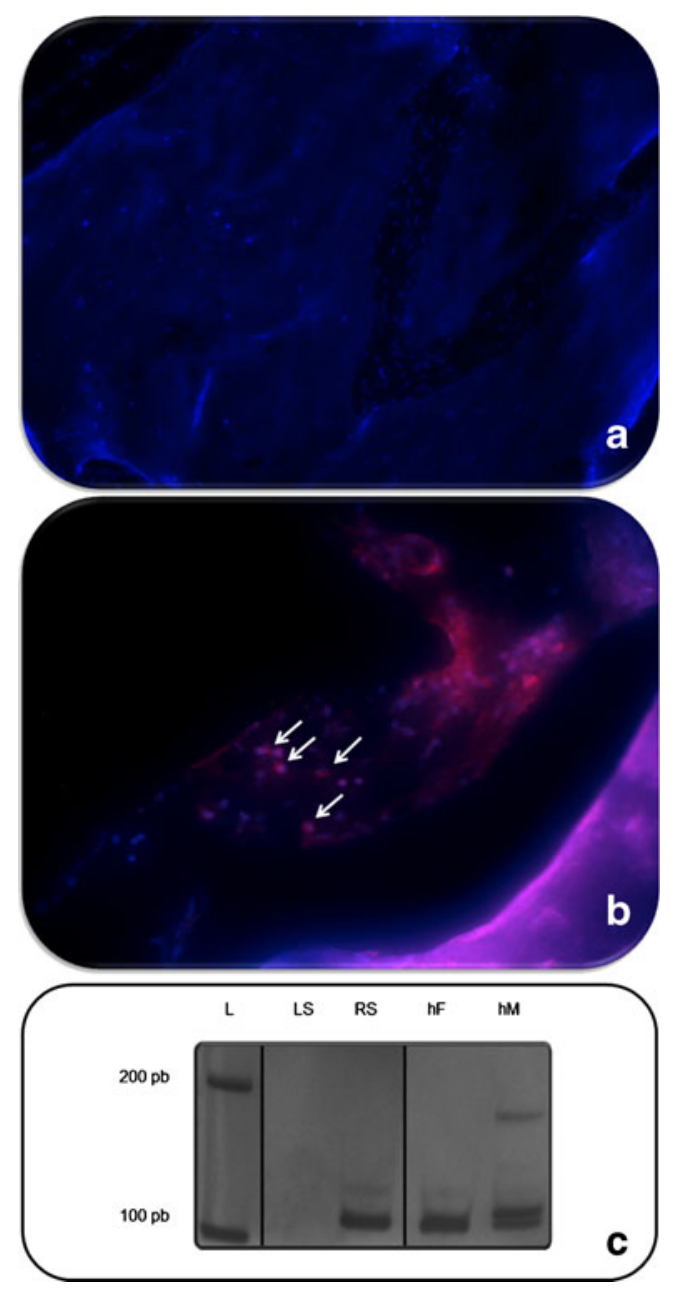

Fig. 4 Human DNA identification. 1 Immunohistochemistry for detection of human nuclei. $a, b$ and $c$ represent the left side (without human stem cells) with DAPI staining (in blue), human nuclei staining (in red) and the overlapping of both pictures, respectively. $a^{\prime}, b^{\prime}$ and $c^{\prime}$ represent the right side (with human stem cells) with DAPI staining (in blue), human nuclei staining (in red) and the overlapping of both pictures, respectively. 2 Human DNA amplification, where: $L$ represents the DNA leader; $L S$ represents the left side of the animal, without human DNA detection; $R S$ represents the right side of the animal with human DNA; $h F$ human female DNA; $h M$ human male DNA
Besides that, human DNA was successfully amplified only in samples extracted from the right side, where the htMSCs had been transplanted (Fig. 4). Thus, these results confirmed that the human cells are part of the new formed bone in the cranial defect.

\section{Discussion}

Strategies for closing bone defects rely on the use of autogenous cancellous bone grafts since they are immunologically inert and potential suppliers of cells with osteoconductive and osteoinductive properties which are required for the bone healing process [14, 15]. However, grafts may result in complications such as donor area morbidity and post-surgical reabsortion $[16,17]$.

The possibility of using stem cells for bone regenerative medicine has opened a new field of investigation. The identification of sources for obtaining multipotent stem cells, in particular through non-invasive procedures, is of utmost interest. In this context, the human fallopian tubes that are regularly discarded during hysterectomy or tubal ligation/ resection procedures may represent a good source of MSCs. Here we report, for the first time, that htMSCs along with CellCeram, present high osteogenic potential, for reconstructing a cranial defect in NIS male Wistar rats. The high expression of mesenchymal and adhesion markers and the lack of expression of hematopoietic or endothelial markers observed in htMSCs lineages are similar to the ones seen in primary MSCs obtained from other sources such as dental pulp, orbicularis oris muscle, adipose or umbilical cord tissue [7-10].

Different types of scaffolds have been used associated with MSCs for bone tissue engineering, such as collagen membrane, hydroxyapatite, hydroxyapatite associated with tricalcium phosphate, and others $[8,9,18,19]$. We chose to associate the htMSCs with CellCeram biomaterial as a scaffold since it was designed to synthetically replicate the hard calcium phosphate extracellular matrix of the bone and display good porosity for cell infiltration and adhesion, which may increase bone regeneration efficiency and promote the rehabilitation of different types of bone defects.

We observed that htMSCs are able to reconstitute a cranial defect in NIS male Wistar rats along with the CellCeram. The newly formed bone tissue in animals analysed 90 days postsurgery is, in part, of human origin, since we were able to amplify its DNA using primers specific for the human amelogenin gene. Additionally, positive staining for human nuclei was exclusively obtained on the right side of the cranial defect.

Although both human and rat cells can form bone, as observed in 90 days postsurgery animals, the contribution of human stem cells was essential because the scaffold alone was not enough to close the cranial defect at least up to 60 days. 
In accordance with our and other researchers previous reports $[8,9,20]$, the present research reinforces that human MSCs transplanted into nonimmunosupressed rats are not rejected and do not elicit any immunological response. The fact that they display the same immunomodulatory properties reported for MSC obtained from different sources enhance the potential application of heterologous htMSCs for bone regeneration $[21,22]$.

Moreover we show that the potential of htMSCs to enhance bone regeneration is comparable to the previously reported with dental pulp and orbicularis oris muscle MSCs $[8,9]$, confirming that fallopian tubes MSCs combined with biomaterials could be a promising additional source for patients with bone diseases or defects.

Finally, although we observed bone formation on both sides of the rat's skull after 90 days, the contribution of human stem cells enhanced significantly the recovery time of the injury.

In short, we demonstrated that htMSCs present excellent osteogenic potential, both in vitro and in vivo. We show, for the first time, that the autologous or heterologous use of htMSCs can successfully enhance bone regeneration in vivo, with potential use for treatment of osteoporosis and bone reconstruction or regeneration.

Acknowledgements We would like to thank entire staff of the Center for Human Genome Studies for technical support and, in particular, the staff of the Laboratory of Stem Cells. Financial Support: FAPESP/CEPID, CNPq and INCT.

Conflict of Interest The authors declare that they have no competing interests.

Authors' contributions TJ, DFB, MRPB and MZ conceived the study. PMP, CEC, MM, LE, MGN and SH provided human tubes from surgical procedures. TJ, DFB and $\mathrm{MZ}$ wrote the manuscript. TJ designed and performed tissue cultures. DFB and BVPA did the surgeries (cranial defects). BVPA and MTM prepared histological blocks and BVPA performed histological analysis. TJ performed flow cytometry and multilineage differentiation. HC prepared scaffolds for electron miscroscopy.

Open Access This article is distributed under the terms of the Creative Commons Attribution Noncommercial License which permits any noncommercial use, distribution, and reproduction in any medium, provided the original author(s) and source are credited.

\section{References}

1. Lewiecki, E. M., Diaz Curiel, M., Borges, J. L., Kung, A., Brandi, M. L., \& Dimai, H. P. (2010). New and emerging therapies for osteoporosis. Journal of Osteoporosis, 2010, 318320.

2. Kawai, M., Mödder, U. I., Khosla, S., \& Rosen, C. J. (2011). Emerging therapeutic opportunities for skeletal restoration. Nature Reviews. Drug Discovery, 10(2), 141-156.

3. Grayson, W. L., Bhumiratana, S., Cannizzaro, C., \& VunjakNovakovic, G. (2011). Bioreactor cultivation of functional bone grafts. Methods in Molecular Biology, 698, 231-241.
4. Bhakoo, K. (2011). In vivo stem cell tracking in neurodegenerative therapies. Expert Opinion on Biological Therapy 7, 911-20.

5. Mohseny, A. B., \& Hogendoorn, P. C. (2001). Concise review: mesenchymal tumors: when stem cells go mad. Stem Cells, 29(3), 397-403.

6. Dominici, M., Le Blanc, K., Mueller, I., et al. (2006). Minimal criteria for defining multipotent mesenchymal stromal cells. The International Society for Cellular Therapy position statement. Cytotherapy, 8, 315-317.

7. Secco, M., Zucconi, E., Vieira, N. M., et al. (2008). Multipotent stem cells from umbilical cord: cord is richer than blood! Stem Cells, 26, 146-150.

8. de Mendonça, C. A., Bueno, D. F., Martins, M. T., et al. (2008). Reconstruction of large cranial defects in nonimmunosuppressed experimental design with human dental pulp stem cells. The Journal of Craniofacial Surgery, 19(1), 204-210.

9. Bueno, D. F., Kerkis, I., Costa, A. M., et al. (2009). New source of muscle-derived stem cells with potential for alveolar bone reconstruction in cleft lip and/or palate patients. Tissue Engineering. Part A, 15(2), 427-435.

10. Vieira, N. M., Brandalise, V., Zucconi, E., et al. (2008). Human multipotent adipose-derived stem cells restore dystrophin expression of Duchenne skeletal-muscle cells in vitro. Biology of the Cell, 100, 231-241.

11. Jazedje, T., Perin, P. M., Czeresnia, C. E., et al. (2009). Human fallopian tube: a new source of multipotent adult mesenchymal stem cells discarded in surgical procedures. Journal of Translational Medicine, 7, 46.

12. Vieira, N. M., Zucconi, E., Bueno, C. R., Jr., et al. (2010). Human multipotent mesenchymal stromal cells from distinct sources show different in vivo potential to differentiate into muscle cells when injected in dystrophic mice. Stem Cell Reviews, 6(4), 560-566.

13. Ray, P. F., Vekemans, M., \& Munnich, A. (2001). Single cell multiplex PCR amplification of five dystrophin gene exons combined with gender determination. Molecular Human Reproduction, 7(5), 489-494.

14. Nwoku, A. L., Al Atel, A., Al Shlash, S., Oluyadi, B. A., \& Ismail, S. (2005). Retrospective analysis of secondary alveolar cleft grafts using iliac of chin bone. The Journal of Craniofacial Surgery, 16(5), 864-868.

15. Rosenstein, A. D., Postak, P. D., \& Greenwald, A. S. (2007). Fixation strength comparison of onlay and inset patellar implants. The Knee, 14(3), 194-197.

16. Younger, E. M., \& Chapman, M. W. (1989). Morbidity at bone graft donor sites. Journal of Orthopaedic Trauma, 3(3), 192-195.

17. Bayerlein, T., Proff, P., Richter, G., Dietze, S., Fanghänel, J., \& Gedrange, T. (2006). The use of ceramic drills on a zirconium oxide basis in bone preparation. Folia Morphologica (Warsz), 65 (1), 72-74.

18. Mankani, M. H., Kuznetsov, S. A., Wolfe, R. M., Marshall, G. W., \& Robey, P. G. (2006). In vivo bone formation by human bone marrow stromal cells: reconstruction of the mouse calvarium and mandible. Stem Cells, 24(9), 2140-2149.

19. Kotobuki, N., Ioku, K., Kawagoe, D., Fujimori, H., Goto, S., \& Ohgushi, H. (2005). Observation of osteogenic differentiation cascade of living mesenchymal stem cells on transparent hydroxyapatite ceramics. Biomaterials, 26(7), 779-785.

20. English, K., French, A., \& Wood, K. J. (2010). Mesenchymal stromal cells: facilitators of successful transplantation? Cell Stem Cell, 7, 431-442.

21. Kaplan, J. M., Youd, M. E., \& Lodie, T. A. (2010). Immunomodulatory activity of mesenchymal stem cells. Current Stem Cell Research and Therapy 6, PMID 21190531.

22. Shi, M., Liu, Z. W., \& Wang, F. S. (2011). Immunomodulatory properties and therapeutic application of mesenchymal stem cells. Clinical and Experimental Immunology 164(1), 1-8. 